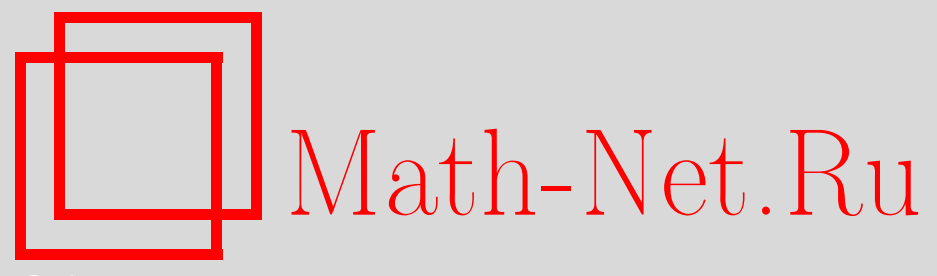

С. Г. Сальников, О максимальной $t$-упаковке полного многодольного графа, Дискрет. матем., 1998, том 10, выпуск 1, 73-81

DOI: https://doi.org/10.4213/dm414

Использование Общероссийского математического портала Math-Net.Ru подразумевает, что вы прочитали и согласны с пользовательским соглашением http://www . mathnet.ru/rus/agreement

Параметры загрузки:

IP : 54.174 .149 .18

26 апреля 2023 г., 14:43:24

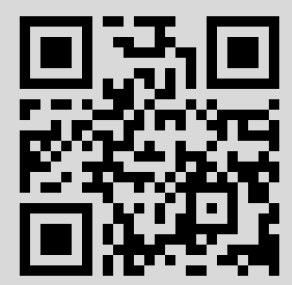




\title{
О максимальной $t$-упаковке полного многодольного графа
}

\author{
(c) 1998 г. С. Г. Сальников
}

\begin{abstract}
Вычисляется максимальное число вершинно независимых копий полного $t$-вершинного графа, которые можно разместить как подграфы в полном многодольном графе с $n$ частями и размерами долей $m_{1}, \ldots, m_{n}$.
\end{abstract}

\section{1. Основные определения}

В данной статье мы следуем стандартным определениям теории графов [1]. В частности, мы рассматриваем графы без кратных ребер и петель. Для графа $G$ через $\nu=\nu(G)$ мы будем обозначать число вершин, а через $\varepsilon(G)$ число его ребер. Два подграфа произвольного (фиксированного) графа $G$ называются вершинно независимыми, если они не имеют общих вершин. Множество попарно вершинно независимых подграфов называется $t$-упаковкой, если каждый из этих подграфов является полным графом на $t$ вершинах. Такую упаковку мы будем называть максимальной $t$-упаковкой произвольного (фиксированного) графа $G$, если не существует $t$ упаковки графа $G$ с большим числом компонент. Число компонент максимальной $t$-упаковки графа $G$ мы будем обозначать через $\psi(t, G)$. Если $t$-упаковка графа $G$ невозможна, то по определенению $\psi(t, G)=0$.

$\Gamma$ раф $G$ называется многодольным, если его множество вершин можно разбить на несколько (не меньше двух) непустых подмножеств таких, что не существует ребер, соединяющих вершины из одной и той же компоненты. Если число долей в многодольном графе равно двум, то граф называется двудольным. Многодольный граф с максимально возможным числом ребер называется полным многодольным графом. Мы будем обозначать $K_{m_{1}, \ldots, m_{n}}$ полный многодольный граф с $n$ частями и с $m_{i}$ вершинами в $i$-й компоненте графа $G, i=1, \ldots, n$. Без потери общности можно считать, что $1 \leqslant m_{1} \leqslant \ldots \leqslant m_{n}$. Всюду далее через $\psi\left(t ; m_{1}, \ldots, m_{n}\right)$ мы будем обозначать величину $\psi\left(t ; K_{m_{1}, \ldots, m_{n}}\right)$. Заметим, что

$$
\nu=\nu(G)=\sum_{i=1}^{n} m_{i} .
$$

Очевидно, что

$$
\begin{aligned}
\psi\left(1 ; m_{1}, \ldots, m_{n}\right) & =\nu, \\
\psi\left(n ; m_{1}, \ldots, m_{n}\right) & =m_{1}, \\
\psi\left(t ; m_{1}, \ldots, m_{n}\right) & =0,
\end{aligned}
$$




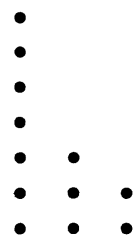

Рис. 1. Точечная диаграмма для разбиения $(7,3,2) \triangleright 12$

если $t>n$.

В [2] в лемме 1 приведена оценка сверху для величины $\psi\left(t ; m_{1}, \ldots, m_{n}\right)$ :

$$
\psi\left(t ; m_{1}, \ldots, m_{n}\right) \leqslant\lfloor\nu / t\rfloor
$$

где $\lfloor x\rfloor$ - наибольшее целое число, меньшее или равное $x$. [3]).

Следующие понятия хорошо известны в теории разбиений чисел (см., например,

Разбиением целого положительного числа $\nu$ называется его представление в виде

$$
\nu=m_{1}+\ldots+m_{n}
$$

где $m_{1} \geqslant m_{2} \geqslant \ldots \geqslant m_{n} \geqslant 1$. Члены суммы называются при этом частями разбиения, а число $n$ слагаемых этой суммы рангом данного разбиения. Для краткости это разбиение числа мы будем обозначать $\left(m_{1}, \ldots, m_{n}\right) \triangleright \nu$.

Точечной диаграммой разбиения $\left(m_{1}, \ldots, m_{n}\right) \triangleright \nu$ называется его представление в виде вершин правильной квадратной решетки, последовательно упорядоченных в виде колонок, так что общее число колонок равно рангу данного разбиения и число точек в $i$-и колонке равно $m_{i}$. Например, на рис. 1 представлена точечная диаграмма для разбиения $(7,3,2) \triangleright 12$.

Очевидно, что для всякого разбиения точечная диаграмма определена однозначно и наоборот, по любой диаграмме такого вида можно однозначно восстановить разбиение, для которого данная диаграмма будет его точечной диаграммой.

Для произвольного (фиксированного) разбиения $\left(m_{1}, \ldots, m_{n}\right) \triangleright \nu$ сопряженным к нему называется разбиение, точечная диаграмма которого получается из точечной диаграммы искомого разбиения симметричным отражением данной диаграммы так, что колонки отражаются в столбцы и наоборот. Так, например, на рис. 2 представлено описанное преобразование для точечной диаграммы разбиения $(7,3,2) \triangleright 12$.

Для сопряженного разбиения используется обозначение $\left(m_{1}, \ldots, m_{n}\right)^{*} \triangleright \nu$. Таким образом, $(7,3,2)^{*} \triangleright 12=(3,3,2,1,1,1,1) \triangleright 12$.

Очевидно, что для произвольного (фиксированного) разбиения $\left(m_{1}, \ldots, m_{n}\right)$ имеет место равенство $\left(\left(m_{1}, \ldots, m_{n}\right)^{*}\right)^{*} \triangleright \nu=\left(m_{1}, \ldots, m_{n}\right) \triangleright \nu$. На множестве разбиений определим слабый суммированный порядок $\prec$ следующим образом:

$$
\left(p_{1}, \ldots, p_{l}\right) \triangleright \nu_{1} \prec\left(m_{1}, \ldots, m_{n}\right) \prec \nu_{2}
$$




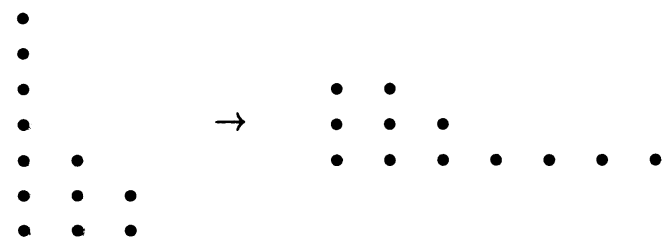

Рис. 2. Построение сопряженного разбиения $(3,3,2,1,1,1,1) \triangleright 12$ для разбиения $(7,3,2) \triangleright 12$

тогда и только тогда, когда для любого целого $i=1, \ldots, l$ имеют место неравенства

$$
\sum_{j=1}^{i} p_{j} \leqslant \sum_{j=1}^{\min (i, n)} m_{j}
$$

Легко проверить, что отношение $\prec$, определенное выше, является отношением частичного порядка на множестве разбиений.

В [2] проводятся исследования экстремальной константы $\psi\left(t ; m_{1}, \ldots, m_{n}\right)$. В частности, эта константа вычислена для случаев $t=1,2, n-1, n$. В статье приводятся также различные оценки снизу и сверху для остальных значений $t$. Цель данной статьи - доказательство следующего результата.

Теорема 1. $\Pi p u n \geqslant t \geqslant 1$

$$
\psi\left(t ; m_{1}, \ldots, m_{n}\right)=\min _{0 \leqslant j \leqslant t-1} \frac{1}{t-j}\left|\sum_{i=1}^{n-j} m_{i}\right|,
$$

$u \psi\left(t ; m_{1}, \ldots, m_{n}\right)=0 n p u t>n \geqslant 1$.

\section{2. Вспомогательные результаты}

Пусть заданы разбиения $\left(p_{1}, \ldots, p_{l}\right) \triangleright \nu_{1}$ и $\left(m_{1}, \ldots, m_{n}\right) \triangleright \nu_{2}$. Обобщим введенное выше понятие $t$-упаковки. Будем говорить, что для данного полного многодольного графа $K_{m_{1}, \ldots, m_{n}}$ существует $\left(p_{1}, \ldots, p_{l}\right)$-упаковка, если в нем можно найти $l$ вершинно независимых подграфов таких, что $i$-й подграф совпадает с $K_{p_{i}}$ для $i=1, \ldots, l$. Заметим, что в случае, когда $p_{1}=p_{2}=\ldots=p_{l}=t$ и $\nu_{1}=\nu_{2}=\nu$, мы получаем обычную $t$-упаковку. Заметим также, что мы можем считать, что $\nu_{1}=\nu_{2}$. Действительно, из определения порядка $\prec$ непосредственно следует, что $\nu_{1} \leqslant \nu_{2}$. Если равенство не выполняется, то дополним набор $\left(p_{1}, \ldots, p_{l}\right)$ должным количеством (а именно $\nu_{2}-\nu_{1}$ ) компонент величины 1 . Ясно, что для полученного набора компонент упаковка возможна тогда и только тогда, когда она возможна для самого набора $\left(p_{1}, \ldots, p_{l}\right)$. Таким образом, будем считать далее, что $\nu_{1}=\nu_{2}=\nu$.

Лемма 1. Для заданных разбиений $\left(p_{1}, \ldots, p_{l}\right) \triangleright \nu u\left(m_{1}, \ldots, m_{n}\right) \triangleright \nu$ mребуемая $\left(p_{1}, \ldots, p_{l}\right)$-упаковка графа $K_{m_{1}, \ldots, m_{n}}$ существует тогда и толъко тогда, когда существует двудолънъй граф, набор степеней первой доли которого совпадает (c 
точностью до порядка) с разбиением $\left(p_{1}, \ldots, p_{l}\right) \triangleright \nu$, а набор степеней второй доли совпадает (с точностью до порядка) с разбиением $\left(m_{1}, \ldots, m_{n}\right) \triangleright \nu$.

Доказательство. Предположим, что указанная в лемме $\left(p_{1}, \ldots, p_{l}\right)$-упаковка графа $K_{m_{1}, \ldots, m_{n}}$ существует. Определим двудольный граф с $l$ вершинами в первой доле и с $m$ вершинами во второй доле следующим образом.

Ребро $(i, j)$ существует тогда и только тогда, когда в $i$-й компоненте данной упаковки, то есть в полном графе $K_{p_{i}}$, в качестве одной из вершин данного графа используется вершина из $j$-й доли графа $K_{m_{1}, \ldots, m_{n}}$. Очевидно, что определенный таким образом двудольный граф обладает описанными в условии леммы свойствами.

Обратно, пусть существует описанный в условии леммы двудольный граф. Построим по данному графу необходимую упаковку полных графов $K_{p_{i}}, i=1, \ldots, l$, в граф $K_{m_{1}, \ldots, m_{n}}$ следующим образом.

Возьмем $m$ наборов вершин будущего многодольного графа так, что в $j$-м наборе вершин имеется $m_{j}$ вершин. Проделаем последовательно для $i=1, \ldots, l$ следующую процедуру: выберем в данном двудольном графе $i$-ю вершину из первой доли; определим номера всех вершин из второй доли, соединенных с данной $i$-й вершиной (таковых, очевидно, будет в точности $p_{i}$ штук); для полученного таким образом набора $j_{1}, \ldots, j_{p_{i}}$ выберем в компонентах с номерами $j_{1}, \ldots, j_{p_{i}}$ упомянутого выше набора вершин по одной (еще не задействованной на этот момент) вершине и соединим все эти вершины ребрами друг с другом, образуя таким образом граф $K_{p_{i}}$.

Очевидно, что таким образом мы построим искомую $\left(p_{1}, \ldots, p_{l}\right)$-упаковку для полного многодольного графа, построенного на упомянутых выше наборах вершин, так как мы задействуем в вышеупомянутой процедуре в точности столько вершин из данной $j$-й компоненты набора вершин, какова степень этой вершины в двудольном графе, то есть $m_{j}$ вершин, и, значит, шаг поиска необходимой свободной вершины на предпоследнем этапе описанной выше процедуры всегда будет успешно осуществлен. Лемма доказана.

Таким образом, как следствие леммы 1 получаем, что основная проблема данной статьи, определение максимальной $t$-упаковки, сводится к поиску двудольного графа с определенным набором вершин в каждой из компонент этого графа. Существенным здесь остается только вопрос о существовании такого двудольного графа с заданным набором степеней в каждой из его долей. Переформулируем данный вопрос следующим образом.

Для заданного двудольного графа с $l$ вершинами в первой компоненте и $n$ вершинами во второй компоненте построим его матрицу инциденций, то есть матрицу $\left\|a_{i j}\right\|$ размера $l \times n$, элементы $a_{i j}$ которой принимают только значения, равные 0 или 1 и $a_{i j}=1$ тогда и только тогда, когда $i$-я вершина первой компоненты соединена с $j$-й вершиной второй компоненты. Ясно, что по заданному двудольному графу его матрица инциденций определяется однозначно с точностью до перестановки ее строк и столбцов. И насборот, по любой $(0,1)$-матрице можно построить (с точностью до изоморфизма графов) соответствующий двудольный граф. Сумма единиц в $i$-й строке данной матрицы равна $p_{i}$, а сумма единиц в $j$-м столбце $m_{j}$. Набор таких сумм, рассматриваемый как вектор с $l$ (или соответственно $m$ ) компонентами, называется вектором строчных (столбцовых) сумм. 
Упомянутый выше вопрос существования формулируется в терминах $(0,1)$ матриц следующим образом.

Для заданных разбиений $\left(p_{1}, \ldots, p_{l}\right) \triangleright \nu$ и $\left(m_{1}, \ldots, m_{n}\right) \triangleright \nu$ определить, существует ли хотя бы одна $(0,1)$-матрица с векторами строчных и столбцовых сумм, совпадающими (с точностью до порядка коміонент) с векторами $\left(p_{1}, \ldots, p_{l}\right)$ и $\left(m_{1}, \ldots, m_{n}\right)$ соответственно.

Проблема, сформулированная выше, хорошо известна в теории блок-схем, и ответ на поставленный вопрос существования требуемой $(0,1)$-матрицы дает переформулированная нами в приведенных выше терминах следующая теорема (см. теорему 11.1 в [4]).

Теорема 2. Для заданнъх разбиений $\left(p_{1}, \ldots, p_{l}\right) \triangleright \nu u\left(m_{1}, \ldots, m_{n}\right) \triangleright \nu$ хотя бъ одна $(0,1)$-матрича с векторами строчных и столбцовых сумм, совпадаюиции (с точностью до порядка компонент) с векторами $\left(p_{1}, \ldots, p_{l}\right) u\left(m_{1}, \ldots, m_{n}\right)$ соответственно, существует тогда и толъко тогда, когда

$$
\left(m_{1}, \ldots, m_{n}\right) \triangleright \nu \prec\left(p_{1}, \ldots, p_{l}\right)^{*} \triangleright \nu .
$$

\section{3. Доказательство теоремы 1}

Пусть $\psi=\psi\left(t ; m_{1}, \ldots, m_{n}\right)$ и $t \leqslant n$. Тогда с учетом упомянутого во вводной части неравенства $\psi\left(t ; m_{1}, \ldots, m_{n}\right) \leqslant\lfloor\nu / t\rfloor$ дополним разбиение $\left(t^{\psi}\right)=(t, \ldots, t)$ с $\psi$ одинаковыми частями размера $t$ частями размера 1 так, что сумма членов данного разбиения станет равна $\nu$ и образуем таким образом разбиение $\left(t^{\psi}, 1^{\nu-t \psi}\right) \triangleright \nu$.

Очевидно, что

$$
\left(t^{\psi}, 1^{\nu-t \psi}\right)^{*} \triangleright \nu=\left(\nu-t \psi+\psi, \psi^{t-1}\right) \triangleright \nu .
$$

Воспользовавшись теоремой 2, выпишем условие

$$
\left(m_{n}, \ldots, m_{1}\right) \triangleright \nu \prec\left(t^{\psi}, 1^{\nu-t \psi}\right)^{*} \triangleright \nu
$$

в явном виде:

$$
\begin{aligned}
m_{n} & \leqslant \nu-\psi t+\psi, \\
m_{n}+m_{n-1} & \leqslant \nu-\psi t+2 \psi, \\
& \ldots \\
m_{n}+m_{n-1}+\ldots+m_{n-t+2} & \leqslant \nu-\psi t+(t-1) \psi, \\
m_{n}+m_{n-1}+\ldots+m_{n-t+1} & \leqslant \nu-\psi t+t \psi=\nu .
\end{aligned}
$$

Отсюда вместе с неравенством $\psi\left(t ; m_{1}, \ldots, m_{n}\right) \leqslant\lfloor\nu / t\rfloor$ получаем, что долхно выполняться неравенство

$$
\psi=\psi\left(t ; m_{1}, \ldots, m_{n}\right) \leqslant \min _{0 \leqslant j \leqslant t-1} \frac{1}{t-j}\left|\sum_{i=1}^{n-j} m_{i}\right| .
$$

Обратно, пусть

$$
\psi_{0}=\min _{0 \leqslant j \leqslant t-1} \frac{1}{t-j}\left|\sum_{i=1}^{n-j} m_{i}\right|
$$


Тогда выполняются все приведенные выше неравенства, в которых вместо $\psi$ необходимо подставить $\psi_{0}$. Но тогда, в соответствии с теоремой 2 существует $(0,1)-$ матрица с соответствующими векторами строчных и столбцовых сумм и, значит, существует соответствующий двудольный граф, но тогда в соответствии с леммой 1 существует $t$-упаковка с $\psi_{0}$ компонентами, откуда получаем (в силу максимальности величины $\left.\psi=\psi\left(t ; m_{1}, \ldots, m_{n}\right)\right)$ неравенство

$$
\psi\left(t ; m_{1}, \ldots, m_{n}\right) \geqslant \psi_{0}=\min _{0 \leqslant j \leqslant t-1} \frac{1}{t-j}\left|\sum_{i=1}^{n-j} m_{i}\right|,
$$

что вместе с доказанным выше обратным неравенством и завершает доказательство теоремы 1.

Как следствие из доказанного основного результата, теоремы 1 , легко получить следующую известную формулу, доказанную в [2].

Следствие 1.

$$
\psi\left(2 ; m_{1}, \ldots, m_{n}\right)=\min \left(\sum_{i=1}^{n-1} m_{i},\left\lfloor\frac{1}{2} \sum_{i=1}^{n} m_{i}\right\rfloor\right)
$$

Следствие 2. Пустъ $m_{n} \leqslant\lfloor\nu / t\rfloor u t \leqslant n$, тогда

$$
\psi\left(t ; m_{1}, \ldots, m_{n}\right)=\lfloor\nu / t\rfloor
$$

Таким образом, справедливо утверждение гипотезы из [2].

\section{4. Реализащии и алгоритм построения максимальной $t$-упаковки}

В данном параграфе предложен эффективный алгоритм для конструктивного построения максимальной $t$-упаковки полного многодольного графа и вычисления экстремальной константы $\psi\left(t ; m_{1}, \ldots, m_{n}\right)$. Алгоритм, предложенный ниже, будет приведен для двудольного графа с заданными наборами степеней вершин его долей. В соответствии с леммой 1 данный алгоритм легко переформулируется далее для задачи построения требуемой $t$-упаковки.

Итак, пусть имеются произвольные (фиксированные) наборы степеней вершин долей полного двудольного графа $\left(p_{1}, \ldots, p_{l}\right) \triangleright \nu$ и $\left(m_{1}, \ldots, m_{n}\right) \triangleright \nu$, рассматриваемые нами как разбиения наперед заданного числа $\nu$, общего количества ребер в данном двудольном графе. Требуется построить (какой-нибудь) двудольный граф, двудольную реализацию, с данным набором степеней вершин его долей. Приводимые ниже результаты позволяют предложить алгоритм построения такого двудольного графа, а значит, как следствие, и любой (наперед заданной и существующей) $t$-упаковки. Эти результаты (и соответствующий алгоритм) аналогичны результатам для так называемых графических последовательностей, когда для заданного набора степеней вершин графа требуется построить граф, реализующий данную последовательность, то есть граф, последовательность степеней вершин которого совпадает (с точностью до порядка) с заданной (графической) последовательностью. 
С результатами в данной области можно ознакомиться, например, в монографии [5] (глава 8 , лемма 45.2 , теоремы $45.1,46.1$, и строящаяся на их основе так называемая l-процедура).

Всюду далее в данном параграфе ребро двудольного графа, соединяющее вершину степени $p_{i}$ из первой доли и вершину степени $m_{j}$, будем обозначать $(i, j)$.

Рассмотрим вначале вопрос о реализации двудольного графа с заданными наборами степеней вершин его долей. Пусть $G$ - двудольный граф, $a, b, c, d-$ четыре его попарно различные вершины, первые две из первой доли и две последние из второй доли, и в графе $G$ имеются ребра $(a, c)$ и $(b, d)$, но отсутствуют ребра $(a, d)$ и $(b, c)$. Тогда будем говорить, что граф $G$ допускает переключение $s=(a c, b d)$. Полученный в результате переключения $s$ граф будем обозначать $s G$; граф $G$ превращается в граф $s G$ в результате удаления ребер $(a, c)$ и $(b, d)$ и присоединения ребер $(a, d)$ и $(b, c)$. Ясно, что данная операция над двудольными графами является обратимой и $s^{-1}=(a d, b c)$. Заметим, что переключение не меняет степеней вершин двудольного графа.

Лемма 2. Для любой вершины $i$ первой доли двудолъного графа $G$ с наборами степеней вершин его долей $\left(p_{1}, \ldots, p_{l}\right) \triangleright \nu u\left(m_{1}, \ldots, m_{n}\right) \triangleright \nu$ сущестєет такая последовательность переключений $s_{1}, \ldots, s_{r}$, что в графе $H=s_{r} \ldots s_{1} G$ эта вершина соединена в точности с вершинами второй доли наиболъшей степени, то есть с вериинами $1,2, \ldots, i$.

Теорема 3. Лґибая двудольная реализация данных наборов степеней вериин двудолъного графа получается из любой другой его двудолъной реализации посредством применения подходящей чепочки переключений.

Доказательства леммы 2 и теоремы 3 почти дословно повторяют доказательства упомянутых выше леммы 45.2 и теоремы 45.1 из [5] и потому здесь не приводятся.

Таким образом, теорема 3 описывает все возможные реализации заданного набора степеней вершин долей двудольного графа и дает способ их пострся яия друг из друга. В общем случае, однако, нахождение числа возможных реализ ций заданных наборов степеней вершин долей двудольного графа представляется сложной задачей.

Перейдем теперь к формулировке алгоритма построения двудольного графа с заданными наборами степеней вершин его долей. Зафиксируем индекс $i$ из первой доли и через $p^{i}$ обозначим разбиение, которое получается из разбиения $\left(p_{1}, \ldots, p_{l}\right) \triangleright \nu$ вычеркиванием $i$-го члена. Далее, через $m^{i}$ обозначим разбиение, которое получается из разбиения $\left(m_{1}, \ldots, m_{n}\right) \triangleright \nu$ в результате уменьшения на единицу каждого из первых $m_{i}$ членов.

Теорема 4. Пусть заданы разбиения $\left(p_{1}, \ldots, p_{l}\right) \triangleright \nu u\left(m_{1}, \ldots, m_{n}\right) \triangleright \nu . \quad E с л u$ для какого-либо индекса $i, 1 \leqslant i \leqslant l$, пара разбиений $p^{i}$ u $m^{i}$ допускает двудолъную реализачию, то и пара $\left(p_{1}, \ldots, p_{l}\right) \triangleright \nu u\left(m_{1}, \ldots, m_{n}\right) \triangleright \nu$ допускает двудольную реализачию. Наоборот, если пара $\left(p_{1}, \ldots, p_{l}\right) \triangleright \nu u\left(m_{1}, \ldots, m_{n}\right) \triangleright \nu$ допускает двудолъную реализачию, то и любая пара $p^{i} u m^{i}, 1 \leqslant i \leqslant l$, допускает двудолъную реализачию.

Доказательство теоремы 4 почти дословно повторяет доказательство теоремы 46.1 из [5] и потому здесь не приводится. 
Изложенные выше результаты позволяют сформулировать алгоритм распознавания наличия двудольной реализации для заданной пары наборов степеней вершин долей двудольного графа и построения одной из возможных реализаций.

Фиксируем наборы $l$ первой доли и $n$ вершин второй доли и проставляем напротив каждой из вершин первой доли метки $p_{1}, \ldots, p_{l}$ и напротив каждой из вершин второй доли метки $m_{1}, \ldots, m_{n}$. Шаги алгоритма заключаются в следующем.

(1) Фиксируем в первой доле вершину с максимальной меткой $M$.

(2) Фиксируем во второй доле первые $M$ максимальных меток.

(3) Проводим ребра, соединяющие выбранную вершину первой доли с выбранными вершинами второй доли.

(4) Меняем метку выбранной вершины первой доли на 0, а метки выбранных вершин второй доли уменьшаем на 1.

(5) Возвращаемся к шагу 1 , если ни одна из меток не стала отрицательной, или прекращаем выполнение алгоритма, если хотя бы одна из меток получает отрицательное значение или все метки получили значение 0.

Как легко видно из приведенных выше результатов, данный алгоритм или построит заданную двудольную реализацию или остановится до присвоения всем вершинам нулевых меток и, значит, выявит, что искомый набор степеней вершин долей не имеет двудольных реализаций. Обоснование данного утверждения почти дословно повторяет соответствующие рассуждения для $l$-процедуры ([5], стр. 212-213) и потому здесь не приводится.

Как частный случай приведем теперь алгоритм построения максимальной $t$ упаковки и соответственно конструктивного вычисления экстремальной константы $\psi\left(t ; m_{1}, \ldots, m_{n}\right)$ при условии, что $n \geqslant t$.

Зафиксируем $n$ параметров с первоначальными значениями $m_{1}, \ldots, m_{n}$, и изобразим для каждого из данных параметров группу вершин (по $m_{i}$ вершин в $i$-й группе, $1 \leqslant i \leqslant n$ ), зафиксируем параметр $t$ и присвоим переменной $\psi$ значение 0 . Далее будем проводить следующие шаги.

(1) Выберем первые $t$ максимальных значений параметров. Уменьшим значение каждого из этих параметров на 1.

(2) Если в результате появится хотя бы один отрицательный параметр, то переходим к следующему этапу. В противном случае в соответствующих группах вершин выбираем по одной (произвольной и еще не задействованной) вершине из каждой группы и строим на данных вершинах полный граф на $t$ вершинах. Значение переменной $\psi$ увеличиваем на единицу и возвращаемся к шагу 1 .

(3) На тот момент, когда алгоритм перейдет на данный шаг, мы будем иметь значение переменной $\psi$, совпадающее со значением максимальной $t$-упаковки, а соответствующие полные $t$-вершинные графы будут образовывать искомую максимальную $t$-упаковку полного многодольного графа на выбранных на начальном этапе алгоритма группах вершин, что и завершает работу алгоритма.

Заметим, что несимметричность результатов, приведенных в данном параграфе, относительно наборов степеней первой и второй доли - явление только кажущееся. 
Переставив наборы степеней вершин долей, можно сформулировать аналогичные несимметричные результаты и достичь тем самым симметрии формулировок результатов данного параграфа.

В заключение заметим, что несмотря на большое сходство приведенных в данном параграфе результатов с соответствующими результатами для графических последовательностей (вследствие чего мы не приводили доказательств результатов), задача построения двудольной реализации заданных наборов степеней вершин долей двудольного графа не сводится, как представляется, к задаче определения существования некоторой графической реализации. Так, например, наличие графической реализации последовательности, получающейся в результате объединения двух данных наборов степеней вершин долей двудольного графа вовсе не гарантирует нам наличие двудольной реализации получившейся графической реализации данного объединенного набора, так как в получившейся графической реализации могут быть ребра между предполагаемыми вершинами одной и той же доли двудольного графа. Конечно, обратное утверждение, что двудольная реализация пары наборов степеней вершин долей является графической реализацией объединения (как множеств) этих последовательностей, верно.

\section{Список литературы}

1. Chartrand G., Lesniak L. Graphs and Digraphs. Wadsworth, Belmont, 1986.

2. Stuart J., Sitton D. Packing complete graphs into complete multipartite graphs. Congressus Numerantium (1996) 118, 3-22.

3. Эндрюс Г. Теория разбиений. Наука, Москва, 1982.

4. Тараканов В. Е. Комбинаторные задачи и $(0,1)$-матрицы. Наука, Москва, 1985.

5. Емеличев В. А., Мельников О. И., Сарванов В. И., Тышкевич Р. И. Лекции по теории графов. Наука, Москва, 1990.

Статья поступила 01.07.97. 